\title{
Implementación tecnologías limpias en unidades mineras ubicadas en la zona aurífera del departamento de Antioquia
}

\section{TEknos}

\author{
Implementation of clean technologies in \\ mining units located in the gold area of \\ the department of Antioquia
}

\author{
Inga Catherine Rodríguez Mur \\ Profesional Universitario (Ingeniera Quimica), \\ irodriguez@corantioquia.gov.co
}

Recibido: 15-feb-17 - Revisado: 30-abr-17

Aceptado: 30-may-17 - Publicado: 30-jul-17

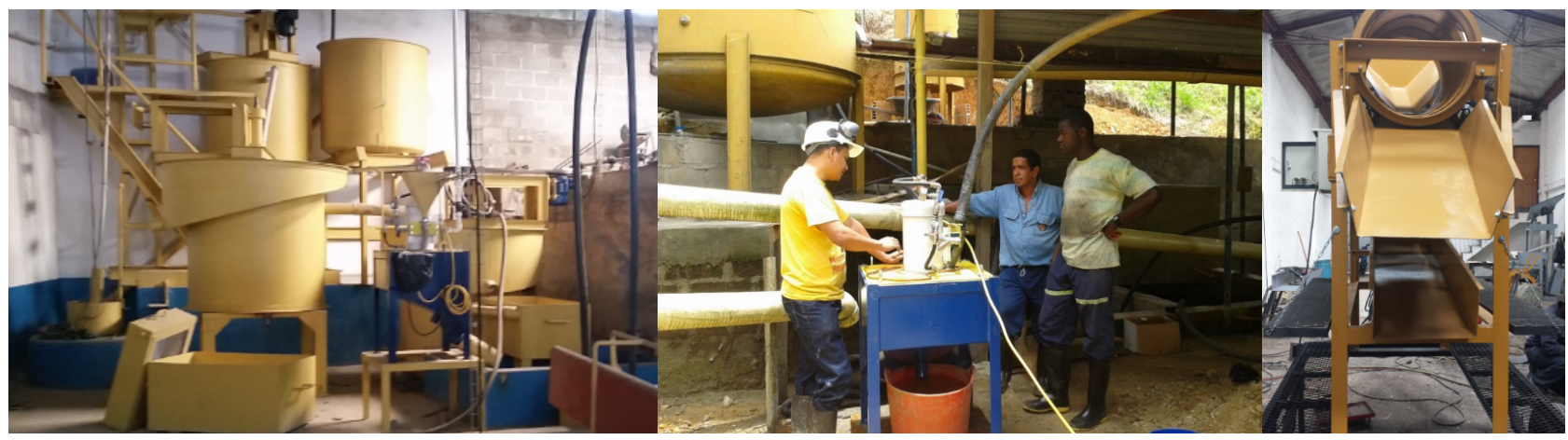

Resumen: Corantioquia, junto a otros actores, viene desarrollando proyectos que buscan reducir el uso del mercurio durante el proceso de extracción del oro libre. Dichos proyectos se han enfocado en incentivar e implementar la aplicación de buenas prácticas y tecnologías limpias mediante programas de capacitación, asesoría técnica, campañas demostrativas de procesos, así como el montaje y puesta en marcha de equipos para tal fin y el monitoreo de mercurio tanto al interior como exterior de establecimientos como entables y compraventa de oro. Con el propósito de lograr este objetivo, se intervinieron múltiples unidades mineras, en especial, en municipios del norte y bajo Cauca de Antioquia. Este documento presenta un análisis de los resultados obtenidos en dichos proyectos; se destaca que la implementación de buenas prácticas y tecnologías limpias genera reducción del uso del mercurio y aumenta el porcentaje de la recuperación del oro, igualmente genera una disminución de los impactos a la salud y al ambiente.

Palabras clave: Mercurio, contaminación, tecnologías limpias, minería aurífera.

Abstract: Corantioquia with other actors has been developing projects to reduce the use of mercury during the gold extraction process. These projects focused on best practices and clean technologies through training programs, technical assistance, demonstration processes and installation of special equipment and monitoring both inside and outside the establishments.

In order to achieve this goal, multiple mining units especially in municipalities in Antioquia were intervened. Results show that implementation of good practices and clean technologies reduce mercury and increase gold recovery. There is also a decrease of impacts on health and the environment. The above results are much more significant with the implementation of clean technologies and comprehensive improvements.

Key words: Mercury, Polution, Clean tecnologies, gold mining. 


\section{INTRODUCCIÓN}

En Colombia, la minería informal y la pequeña y mediana minería ocupan una gran parte de la producción de oro del país. Dentro de esta producción, el departamento de Antioquia representa el territorio con mayor porcentaje de mineral explotado y presenta, a la vez, un elevado potencial aurífero para futuras explotaciones. Se destaca, además, que cada vez es mayor el número de solicitudes para proyectos mineros que recibe el departamento (Contraloría General de la República, 2013).

La extracción de oro en la minería artesanal y a pequeña escala del país se ha desarrollado tradicionalmente usando mercurio para separar el oro libre del material que no tiene interés económico; este proceso es conocido como amalgamación. Una de las principales causas de una amalgamación ineficiente de oro es la presencia de minerales acompañantes o impurezas que impiden una reacción perfecta entre el metal y el mercurio (UPME, 2007). Las medidas para minimizar estas contaminaciones y aumentar la recuperación de oro deben de estar orientadas a mejorar los diseños y las eficiencias de los procesos de beneficio con la implementación de métodos alternativos como la flotación de minerales y la concentración gravimétrica. Igualmente, y encaminados a un desarrollo sostenible, dichos métodos desestiman el uso del mercurio en el proceso de extracción del oro libre (Corantioquia, Gobernación de Antioquia \& Universidad Nacional de Colombia, 2015).

El uso de mercurio $(\mathrm{Hg})$ en la actividad minera ha provocado una alta contaminación en el aire, el agua y el suelo (Argüello, Cantos y Viteri 2011). Esto ocurre principalmente en las zonas donde se beneficia dicho material, debido a las características del mercurio como su alta densidad, baja biodegradabilidad y evaporación a temperatura ambiente; lo anterior genera que la contaminación se disperse a otros territorios por medio del agua y el aire. Adicionalmente, la salud de las personas que trabajan en las plantas de beneficio de oro también se ve afectada debido a la alta toxicidad de dicho elemento. Teniendo en cuenta las anteriores consideraciones, la Corporación Autónoma Regional del Centro de Antioquia (Corantioquia) desde hace varios años, suma esfuerzos en este tema con distintas entidades: la Organización de Naciones Unidas para el Desarrollo Industrial (ONUDI), el Ministerio de Medio Ambiente y Desarrollo Sostenible (MADS), la Universidad Nacional de Colombia sede Medellín, la Universidad de Antioquia y la Gobernación de Antioquia.

El trabajo realizado busca conocer el estado de los municipios que desarrollan minería aurífera en Antioquia y fortalecer la implementación de tecnologías limpias que ayuden a reducir la cantidad de emisiones contaminantes y residuos, además de aumentar la productividad y por ende, los ingresos; así, se espera mejorar la calidad de vida de la población minera.

Para el desarrollo de los proyectos, se priorizaron municipios mineros dentro del departamento de Antioquia donde la actividad minera es muy representativa (Fig. 1). En este documento, se presentan los resultados más significativos de dos convenios. El Convenio 1 se desarrolló con la Gobernación de Antioquia y la Universidad de Antioquia, enfocado en el diagnóstico a nivel sanitario y ambiental y la asesoría y capacitación técnica para lograr mejoras en las actividades (Corantioquia, Gobernación de Antioquia \& Universidad de Antioquia, 2013). El convenio 2 se llevó a cabo con la Gobernación de Antioquia y la Universidad Nacional sede Medellín, y estuvo enfocado a la implementación de tecnologías limpias para la reducción del uso del mercurio, con capacitación y demostraciones técnicas (Corantioquia et al., 2015). 


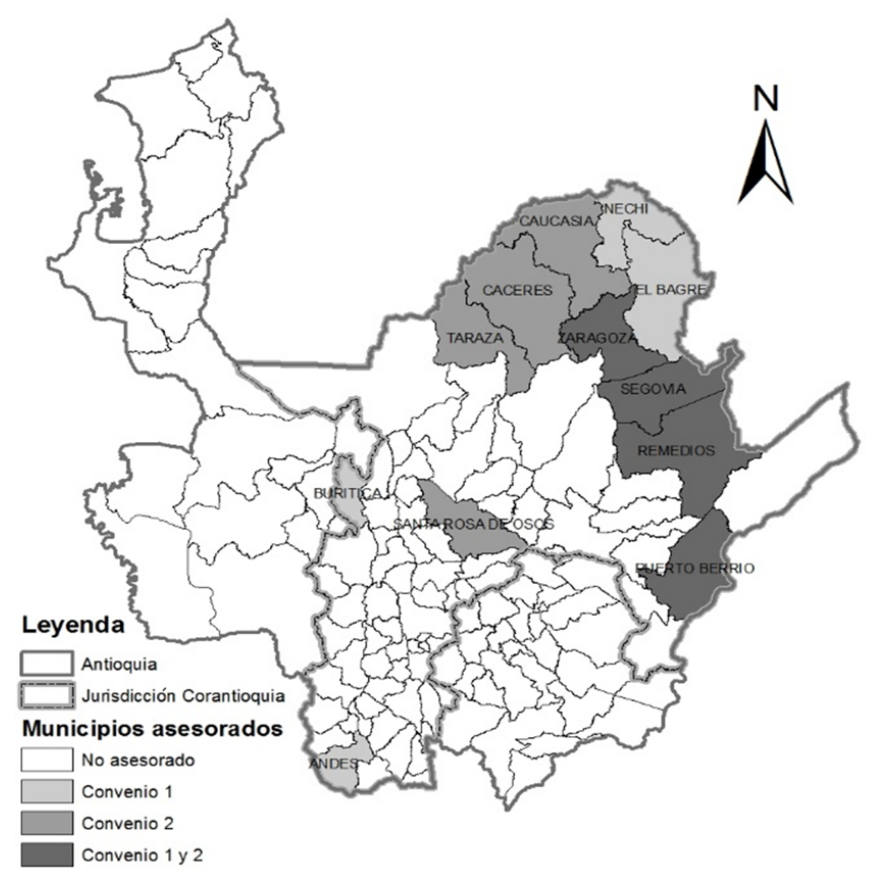

Fig. 1. Municipios seleccionados para el desarrollo de los convenios de mejora a la actividad minera.

\section{MATERIALES Y MÉTODOS}

En el Convenio 1 de Corantioquia con la Gobernación de Antioquia y la Universidad de Antioquia, realizado entre el 2013 y 2014, se trabajó en la caracterización de variables locativas, ambientales, de salud en el trabajo y de los procesos de los entables y compras de oro. Adicionalmente, se trabajó en la medición ambiental de vapores de mercurio, la caracterización del mineral, la elaboración de planes de vigilancia epidemiológica y el análisis de muestras biológicas del personal de los entables, de los establecimientos de compraventa de oro y de los vecinos de la zona.

En el Convenio 2 de Corantioquia con la Universidad Nacional sede Medellín y la Gobernación de Antioquia, realizado entre el 2014 y 2015, enfocado en la implementación de tecnologías limpias para la reducción del uso del mercurio, se trabajó en el diagnóstico de las minas y las plantas de beneficio de oro y la toma y caracterización de muestras del mineral en las diferentes partes del proceso mediante análisis mineralógico, fisicoquímico y metalúrgico.

A partir de dichos resultados, se propusieron modelos de cambio de proceso en las minas seleccionadas y se compraron los equipos requeridos para el mejoramiento del proceso como hidrociclones, bombas de sólidos, concentradores centrífugos, trommel, muflas, bateas mecánicas y tela recuperadora sintética.

\section{RESULTADOS}

\subsection{Convenio 1. Corantioquia, Universidad de Antioquia y Gobernación de Antioquia}

En este convenio, se asesoraron 81 entables mineros y 90 establecimientos de compraventa de oro, para un total de 1440 personas asesoradas en ocho municipios (Tabla 1). De este convenio, se destacan los resultados presentados a continuación. 
Tabla 1. Municipios, entables, compras de oro y población asesorada.

\begin{tabular}{|l|c|c|c|}
\hline \multicolumn{1}{|c|}{ MUNICIPIO } & ENTABLES & $\begin{array}{c}\text { ESTABLECIMIENTOS DE } \\
\text { COMPRAVENTA DE ORO }\end{array}$ & $\begin{array}{c}\text { POBLACIÓN } \\
\text { (\# Personas) }\end{array}$ \\
\hline Nechí & 10 & 8 & 134 \\
\hline Bagre & 0 & 22 & 213 \\
\hline Zaragoza & 8 & 17 & 156 \\
\hline Segovia & 23 & 23 & 314 \\
\hline Remedios & 9 & 5 & 182 \\
\hline Andes & 10 & 4 & 143 \\
\hline Buriticá & 9 & 6 & 50 \\
\hline Puerto Berrío & 12 & 5 & 248 \\
\hline Total & $\mathbf{8 1}$ & $\mathbf{9 0}$ & $\mathbf{1 4 4 0}$ \\
\hline
\end{tabular}

Fuente: Corantioquia et al., 2013.

\subsubsection{Monitoreo ambiental de mercurio} Monitoreo ambiental interno de mercurio: Del grupo de entables y compras de oro estudiadas, se seleccionaron diversos establecimientos a los cuales se realizó medición ambiental de mercurio con un equipo marca Jerome modelo $431 X$, con un rango de lectura de 0,001 a 0,999 mg/m $\mathrm{m}^{3} \mathrm{Hg}$ y con Sensibilidad de $0,003 \mathrm{mg} / \mathrm{m} 3 \mathrm{Hg}$. En total, se realizaron 327 mediciones ambientales al interior de los establecimientos, de las cuales, un alto porcentaje mostraron valores superiores a $0,025 \mathrm{mg} \mathrm{Hg} / \mathrm{m}^{3}$, límite establecido por la American Conference of Governmental Industrial Hygienists (ACGIH).

Monitoreo ambiental externo de mercurio: Teniendo en cuenta la necesidad de determinar el impacto de la actividad minera en la calidad del aire, se efectuaron 84 mediciones ambientales externas, utilizando el equipo Lumex RA-915. Algunos resultados para los municipios de Segovia y El Bagre se muestran en la Tabla 2.

Tabla 2. Resultados monitoreo mercurio ambiental externo en $\mu \mathrm{g} \mathrm{Hg} / \mathrm{m}^{3}$.

\begin{tabular}{|l|c|c|c|}
\hline Lugares de medición & Marzo de 2010 & Marzo 2011 & Noviembre 2012 \\
\hline Algunas calles de Segovia: & 13,6 & 6,0 & 2,85 \\
\hline Algunas calles de El Bagre & 11,3 & 5,8 & - \\
\hline Interior Alcaldía Segovia & 8,0 & 0 & - \\
\hline Cerca Alcaldía Bagre & 12,0 & 5,0 & - \\
\hline Colegio Diocesano & 1,5 & 0 & 0,81 \\
\hline Centro Comercial Segovia & 1,0 & 3,3 & 0,50 \\
\hline Calles frente a entables, Segovia & 60,0 & $5,0-8,0$ & 3,38 \\
\hline
\end{tabular}

Fuente: Corantioquia et al., 2013.

\subsubsection{Monitoreo biológico de mercurio (personas)} Con el fin de evaluar las afectaciones a la salud de los mineros y de la población en general, , se tomaron 1141 muestras para determinar el nivel de mercurio. Se encontró que un 27 \% (312 personas) presenta intoxicación por mercurio; sus resultados son superiores a $35 \mu \mathrm{g} \mathrm{Hg} / \mathrm{gr}$ de creatinina (Mercuriados, 2016).

En muchos de los entables se lograron mejoras a partir 
de la aplicación de buenas prácticas y la implementación de tecnologías limpias sencillas. En la Tabla 3 se muestran las mejoras logradas más sobresalientes, resultado de la asesoría técnica aportada a los entables mineros.
Todas son buenas prácticas mineras, a excepción de la quema de la amalgama utilizando retortas o cubículos con destilador, la cual corresponde a la implementación de tecnologías limpias.

\subsubsection{Asesoría técnica a las empresas}

Tabla 3. Mejoras logradas a raíz de la asesoría técnica a los entables mineros

\begin{tabular}{|l|c|}
\hline \multicolumn{1}{|c|}{ Mejoras logradas } & Número de entables \\
\hline Disminución del consumo de mercurio por coco (de 3,5 Oz a 1,5 Oz por coco) & 45 \\
\hline Concentración del mineral antes de amalgamar & 23 \\
\hline Remolienda sin mercurio & 22 \\
\hline Quema de la amalgama utilizando retortas o cubículos con destilador & 20 \\
\hline Evaluación cambio de proceso de amalgamación a cianuración & 7 \\
\hline Mejoramiento de las instalaciones locativas & 25 \\
\hline Tratamiento de los residuos del proceso & 2 \\
\hline Uso de equipos de protección personal & 40 \\
\hline Sensibilización frente a la toxicidad del mercurio y la contaminación que genera & 81 \\
\hline
\end{tabular}

Fuente: Corantioquia et al., 2013.

Tabla 4. Mejoras logradas a raíz de la asesoría técnica en los establecimientos de compraventa de oro.

\begin{tabular}{|l|c|}
\hline \multicolumn{1}{|c|}{ Mejoras logradas } & $\begin{array}{c}\text { Número de } \\
\text { establecimientos }\end{array}$ \\
\hline Empleo de chimeneas con filtros de carbón y otros & 10 \\
\hline Quema de la amalgama en retortas o cubículos con destilador & 8 \\
\hline Recuperación del mercurio, activación y reúso en el proceso & 35 \\
\hline Separación de la actividad de quema de amalgama de otras actividades económicas & 5 \\
\hline Almacenamiento adecuado de mercurio para evitar pérdidas por volatilización & 40 \\
\hline Uso de equipos de protección personal & 50 \\
\hline Mejoramiento de condiciones locativas & 5 \\
\hline Sensibilización frente a la toxicidad del mercurio y la contaminación que genera & 90 \\
\hline
\end{tabular}

Fuente: Corantioquia et al., 2013.

En cuanto a los establecimientos de compraventa de oro, se destacan las buenas prácticas y tecnologías limpias que se presentan en la Tabla 4.

\subsubsection{Otros}

Adicional a las mejoras en los entables y en los establecimientos de compraventa de oro, se trabajó con las administraciones municipales con la finalidad de avanzar con los directores locales de salud en la construcción de los planes de vigilancia epidemiológica.

Adicionalmente, se construyeron mapas con los niveles de concentración del mercurio y una cartilla de "Manejo integral del mercurio: un enfoque social y de desarrollo humano» (Corantioquia, Gobernación de Antioquia \& Universidad de Antioquia, 2014). 


\subsection{Convenio con Universidad Nacional y Gobernación}

Para este proyecto, se trabajó con 21 unidades mineras ubicadas en los municipios de Antioquia presentados en la Fig. 1. Todas las unidades fueron caracterizadas mineralógicamente y asesoradas por profesionales idóneos. Diez unidades fueron dotadas con equipos de tecnología limpia.

El proceso de caracterización mineralógica que se realizó a las 21 unidades mineras consistió en hacer diferentes pruebas de laboratorio como ensayos
Knelson, ensayo al fuego, análisis granulométrico y análisis mineralógico. A partir de dichos resultados, se inició la optimización del proceso de beneficio de oro atendiendo las particularidades de cada una de las unidades mineras.

Posterior a la caracterización mineralógica y las visitas técnicas, se diseñó una planta de beneficio para extracción de oro en donde no se utiliza mercurio; en ella, se aplica el circuito de beneficio de oro que se presenta en la Fig. 2.

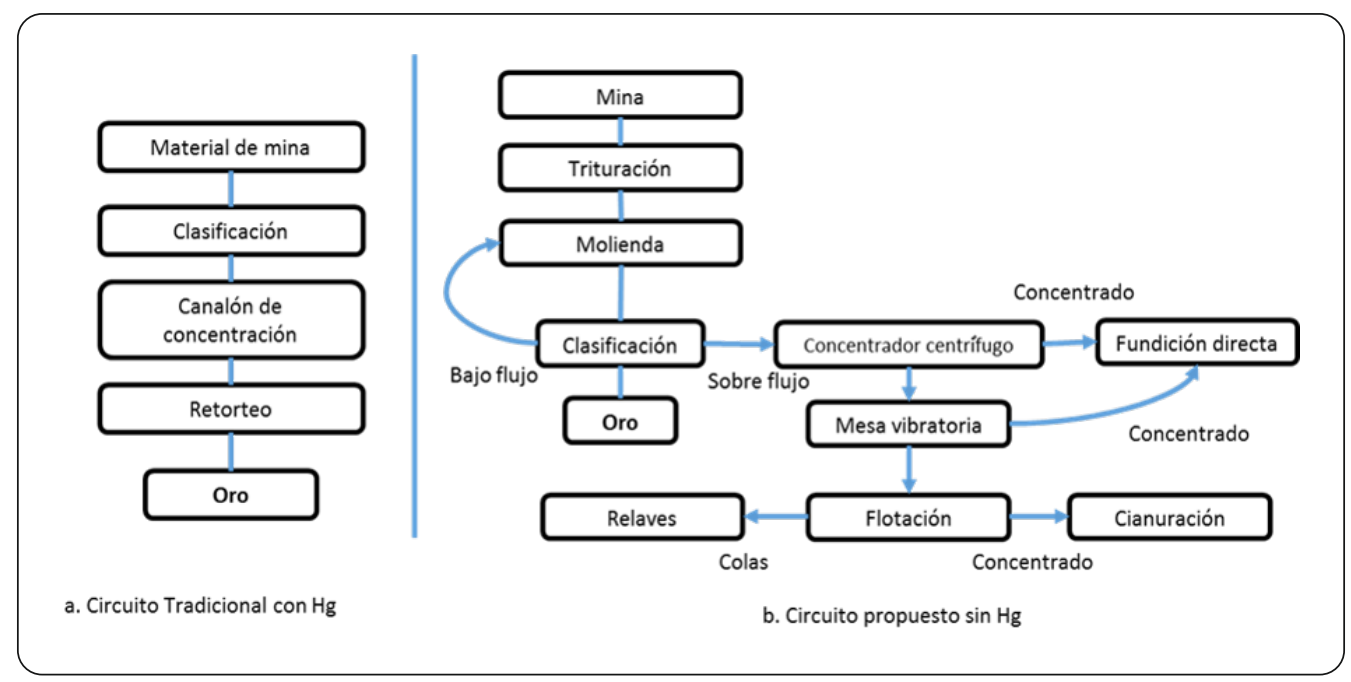

Fig. 2. Circuitos de beneficio del oro: a. Circuito tradicional con mercurio; b. Circuito propuesto sin mercurio. Fuente: Corantioquia et al., 2015.

De acuerdo a la propuesta de mejoramiento tecnológico en las diferentes plantas de extracción de oro seleccionadas, según los resultados de la caracterización de las mismas, se efectuó la compra de equipos y se continuó trabajando en la instalación y puesta a punto de los procesos. Dentro de los equipos comprados, se encuentran: concentradores centrífugos, bombas de sólidos, muflas, hidrociclones, balanzas, molinos de bolas, bateas mecánicas, trommel, mesas de concentración, trommel con canalón en Z, hornos.
Posterior a la puesta a punto de los procesos, se realizaron talleres sobre tecnologías limpias como estrategia en la disminución de uso de mercurio y mejoramiento de la productividad del beneficio del oro a la población minera de los diferentes municipios. En 2015, se logró cuantificar una disminución de uso del mercurio en un $60 \%$ y un aumento en la recuperación de oro del orden, también del $60 \%$ en las unidades mineras intervenidas. Además, se identificó una disminución del 70 \% del consumo de agua mediante recirculación al proceso. Asimismo, se han 
mejorado la calidad de los vertimientos por implementación de tratamientos previos.

\section{DISCUSIÓN DE RESULTADOS}

De las 327 mediciones de mercurio al interior de los establecimientos, un alto porcentaje arrojó valores superiores a $0,025 \mathrm{mg} \mathrm{Hg} / \mathrm{m} 3$, límite establecido por la American Conference of Governmental Industrial Hygienists $(\mathrm{ACGIH})$. Esto indica una alta exposición al mercurio por parte de los trabajadores en los entables y los establecimientos de compraventa de oro. Lo anterior cuenta con un agravante adicional: la evidencia de un bajo uso de equipos de protección personal. Esto es coherente con el resultado de 312 personas que presentaron intoxicación por mercurio, es decir, un $27,3 \%$ del total a las que se realizaron muestras de sangre.

De acuerdo a los resultados del monitoreo de mercurio en el exterior en los diferentes municipios, en la Tabla 2 se observa que casi todas las mediciones superan el nivel máximo permisible para el mercurio como contaminante no convencional con efecto carcinogénico, el cual es de $1 \mu \mathrm{g} \mathrm{Hg} / \mathrm{m}^{3}$ de aire en un tiempo de exposición anual (Ministerio de Medio Ambiente, 2010). Se observa que con el trabajo realizado durante los proyectos, se logró una disminución altamente considerable de concentraciones de mercurio en la calidad del aire; se aclara que las mediciones corresponden al inicio del periodo de exposición y por tanto, es incorrecto inferir que se está ante un evento de exposición anual.

Dentro de las buenas prácticas implementadas en los entables intervenidos, se destaca la reducción del consumo de mercurio por coco, la concentración del mineral antes de amalgamar, la remolienda sin uso de mercurio y la quema de la amalgama utilizando retortas o cubículos con destilador. Tales prácticas aportan considerablemente a los resultados con relación a la disminución de mercurio en el proceso. Para el caso de los establecimientos de compraventa de oro, se resalta el empleo de chimeneas con filtros de carbón, la quema de la amalgama en retortas o cubículos con destilador y recuperación del mercurio y la activación y reúso en el proceso.

En general, la pequeña y la mediana minería en el departamento de Antioquia lleva a cabo vertimientos de las aguas residuales del proceso a las fuentes de agua cercana. Mediante los proyectos realizados, se implementaron lagunas naturales en los predios de las unidades mineras, con el fin de usar el suelo como filtrante de las aguas residuales y poder reusar lo que antes era un residuo como materia prima que entra nuevamente al proceso.

Con esto se logró una disminución del 70 \% del consumo de agua en las unidades mineras intervenidas. Lo anterior tiene un beneficio adicional y es la disminución en el pago de la tasa retributiva que se debe pagar a la Autoridad Ambiental por el vertimiento de las aguas residuales.

El modelo de planta de beneficio de oro que propone el proyecto está diseñado para que no se use mercurio en el proceso. A través de los resultados obtenidos, se evidencia una disminución del uso de mercurio y el aumento de la recuperación del oro a medida que las unidades mineras van mejorando las etapas del proceso; cuantas más etapas mejoradas haya, mejores son los porcentajes de disminución de mercurio y de recuperación de oro.

\section{CONCLUSIONES}

Los municipios seleccionados en estos convenios corresponden a municipios donde la minería es una de las actividades económicas más representativas.

La mediana y pequeña minería de los municipios intervenidos es una actividad artesanal con bajo o nulo uso de tecnologías y escasa implementación de las normas relacionadas con la seguridad y salud en el trabajo. 
Existe una alta contaminación a la salud y al ambiente por la utilización de mercurio en la pequeña y mediana minería de los municipios intervenidos.

Las buenas prácticas ambientales en entables y compras de oro aportan significativamente a la reducción del uso de mercurio y a la optimización del proceso; resaltamos que la implementación de la mayoría de estas no requiere altas inversiones.

La implementación de tecnologías limpias, principalmente en entables, representa mayor aporte en la disminución de la utilización del mercurio, el aumento de la recuperación del oro y por ende, en menores impactos a la salud y el ambiente.

La disminución del uso de mercurio y el aumento en la eficiencia de recuperación de oro se ve relacionada con la implementación de las mejoras en todas las etapas del proceso de beneficio del oro; es decir, cuanto más integral, mejores resultados.

Se recomienda continuar este tipo de proyectos, incluyendo el seguimiento a las unidades mineras intervenidas.

AGRADECIMIENTOS: Se agradece a Corantioquia y a las instituciones y personas que participaron en los convenios, de los cuales se obtuvieron estos resultados.

\section{REFERENCIAS}

Argüello Mejía, A.; Cantos Aguirre, E. \& Viteri Moya, J., (2011). Riesgos antrópicos generados por la actividad minera. Revista Letras Verdes, (11), 53-63. Recuperado de:

http://www.flacsoandes.org/letrasverdes.

Contraloría General de la República (2013). Minería en Colombia: Fundamentos para superar el modelo extractivista. Director Luis Jorge Garay Salamanca 209 p.

Corantioquia, Gobernación de Antioquia \& Universidad de Antioquia (2013). Diagnóstico a nivel sanitario y ambiental y asesoría y capacitación técnica para lograr mejoras en las actividades mineras. Informe Final, Convenio Interadministrativo. Medellín: Corantioquia.

Corantioquia, Gobernación de Antioquia \& Universidad de Antioquia (2014). Cartilla Manejo integral del mercurio: un enfoque social y de desarrollo humano. $23 \mathrm{p}$.

Corantioquia, Gobernación de Antioquia \& Universidad Nacional de Colombia Sede Medellín (2015). Tecnologías limpias en unidades mineras. Medellín, Colombia. $21 \mathrm{p}$.

Mercuriados (2016). Límites "admisibles" de mercurio. Recuperado de:

http://www.mercuriados.org/es/pag115.

Ministerio de Medio Ambiente, Vivienda y Desarrollo Territorial (2010). Resolución 610 de 2010. Bogotá, Colombia.

Unidad de Planeación Minero Energética (UPME) (2007). Producción más limpia en la minería de oro en Colombia mercurio, cianuro y otras sustancias. Recuperado de http://upme.gov.co/Docs/Mineria_Limpia.pdf 